\title{
Study on the Classification of Pulse Signal Based on the BP Neural Network
}

\author{
Shaohua Wang ${ }^{1}$, Jianli Jiang², Xiaobing Lu ${ }^{3}$ \\ ${ }^{1}$ The Second People's Hospital of Yichang, Yichang, China \\ ${ }^{2}$ The First People's Hospital of Yichang, Yichang, China \\ ${ }^{3}$ The Institute of Mechanics, Chinese Academy of Sciences, Beijing, China \\ Email:xblu@imech.ac.cn
}

How to cite this paper: Wang, S.H., Jiang, J.L. and Lu, X.B. (2020) Study on the Classification of Pulse Signal Based on the BP Neural Network. Journal of Biosciences and Medicines, 8, 104-112.

https://doi.org/10.4236/jbm.2020.85010

Received: March 23, 2020

Accepted: May 5, 2020

Published: May 8, 2020

Copyright (c) 2020 by author(s) and Scientific Research Publishing Inc. This work is licensed under the Creative Commons Attribution International License (CC BY 4.0).

http://creativecommons.org/licenses/by/4.0/

\begin{abstract}
The objectification of the pulse signal analysis is a practical problem. The classification of the pulse signal is studied based on the BP neural network. It is first analyzed how to select the characteristic factors of the pulse signal. Then the method of nondimensionalization/normalization on the pulse signal is presented to preprocess the characteristic factors. The classification of the pulse signal and the effects of the selection of characteristic factors are studied by using the normalized data and BP neural network. It is shown that nondimensionalization/normalization of the data is in favor of the training and forecasting of the network. The selection of characteristic factors affects the accuracy of forecasting obviously. The results of forecasting by selection of 8,6 and 4 factors respectively show that the less the factors are, the worse the effects are.
\end{abstract}

\section{Keywords}

BP Neural Network, Pulse Signal, Classification, Dimensional Analysis

\section{Introduction}

Traditional Chinese medicine doctors diagnose a disease in four ways: observation, listening, interrogation and pulse-taking. Pulse-taking is used to diagnose a disease by touching and feeling the pulse manifestation on a human body's surface. From the viewpoint of modern medicine, Pulse manifestation comes in fact from the impulse when the blood flows through the elastic blood vessel. Since the blood flows through all parts of the body, the pulse manifestation contains much information of the body including diseases. The impulse of blood vessel can be felt in the superficial skin such as radial artery, which is called the pulse-taking 
in the traditional Chinese medicine. Though the pulse-taking can diagnose diseases without pain and invasion, this kind of diagnose is related very closely to the clinical experiences of the doctor. In other words, the human factors affect the diagnosis results greatly. Therefore, many studies are focused on the objectification of pulse manifestation to realize the modernization of the pulse-taking as early as possible [1].

Kinds of instruments for pulse diagnosis with different functions and characteristics have been researched and developed and many data have been collected. Based on these data, studies on the pulse manifestation analysis and identification have been carried out, including the feature extraction and identification of single factor and multiple factors. The methods of statistical regression and artificial intelligence are applied in the analysis [2]. The former studies are mainly concentrated on two aspects: one is on the selection of pulse characteristic values and the other is the study of the classification approaches. The former is the base of the later. Clustering analysis is one of the early methods adopted in the pulse classification. This method determines the degree of similarity first by the distance formula; then the data set is divided into several clusters according to the degree of similarity. The data in the same cluster have the close degree of similarity [3]. The method of training and learning by samples is applied recently. The typical one is the classifier based on neural network which is trained by the clinical data of pulse manifestation to obtain the classifier and then is used in the classification of the newly collected pulse manifestation [4] [5]. Some are the combination of several methods, such as fuzzy analysis is combined with artificial neural network method, wavelet is combined with artificial neural network method, etc. [1] [6] [7] [8]. However, the identification of pulse manifestation is still in the stage of exploration. The selection of the characteristics of pulse has been studied and many methods have been provided. Some methods choose the characteristics of time domain or frequency domain such as cardiac cycle or frequency, the maximum pressure and tide wave; some choose the maximum power spectrum and cepstrum as the characteristics, etc.

On the above viewpoint, the selection of the pulse characteristics and the normalization method are investigated first. Then the BP neural network is used to study the identification of pulse manifestation.

\section{Introduction of BP Artificial Neural Network}

A BP [9] network model is consisted of input layer, middle layers and output layer. The dependent variables are input in the input layer. The data outcome from the output layer are the independent variables. The layer locates between the input and output layers are called the middle/hidden layers. The factors of the model are built by learning from the history data. The relations between dependent variables and independent variables may be determined at one time and the results are influenced few even if some dependent or independent data are lost. 
The neuron is the basic unit and the processing element. A neuron, as shown in Figure 1, has many inputs $X_{i}(n-1)$ come from the neurons in the $(n-1)^{\text {th }}$ layer, but only a single output $X_{j}(n)$ that carries its information to the neurons in the $(n+1)^{\text {th }}$ layer. An adjustable weight, $W_{i j}(n)$, representing the connecting strength, lies between the $f^{\text {th }}$ input branch in the $i^{\text {th }}$ layer and the $I^{\text {th }}$ neuron in the $(n-1)^{\text {th }}$ layer. The basic function (net sum) of a neuron is to sum up its inputs and by means of the transfer function to produce an output [10]. Usually, an internal threshold $t$ is introduced and subtracted from the sum. Mathematically, the net sum net, $(n)$ of the $f^{\text {th }}$ neuron in the $n^{\text {th }}$ layer can be expressed as

$$
n e t_{j}^{n}=\sum_{i} W_{i j}^{n} x_{i}^{n-1}-t_{j}^{n}
$$

Then the output value $x_{j}^{n}$ can be obtained as

$$
x_{j}^{n}=f\left(n e t_{j}^{n}\right)=\frac{\theta_{j}^{n}}{1+e^{-\beta_{j}^{n} n e t_{j}^{n}}}
$$

in which $f($.$) is the transfer function which has several forms, such as the hyper-$ bolic tangent, step, or sigmoid function. The sigmoid function is the most common in practical application.

The BP neural network is characterized by gaining possession of hidden layers. A BP neural network without a hidden layer is adequate to describe the system of the linearized relationship between inputs and outputs. Generally, a BP network with one hidden layer is enough for most applications. Therefore, the modified BP network model is adopted here which is a three-layer network with input layer, middle layer and output layer (Figure 1).

In the BP learning scheme, the calculated outputs in the output layer, $x_{j}^{q}$, are compared with the desired outputs, $d_{p}$ to find the error, before the error signals are propagated backward through the network. The error function $\mathrm{E}$ is defined as [11]

$$
E=\frac{1}{2} \sum_{j=1}^{p}\left(d_{j}-x_{j}^{q}\right)^{2}
$$

where $q$ is the total number of layers in the network and $p$ is the total number of the output neurons. The learning process is to adjust the learning parameters, $W, t, \beta$ and $\theta$, so that the error can be minimized and the mapping between inputs and outputs can be realized. In order to accomplish this, gradient descent technique is applied to calculate the gradient of the error with respect to each learning parameter. Then the learning parameters are changed and adjusted in the direction of the steepest descent of the error. For example, the adjustment of the weight parameter is expressed as

$$
\Delta W_{i j}^{n}(T+1)=-\eta \frac{\Delta E}{\Delta W_{i j}^{n}}
$$

where $\eta$ is the learning rate and $T$ is the iteration. There are many methods to update the parameters of the networks. Generally, the pattern learning method is commonly used for its faster convergence speed. This method updates the parameters of the network whenever the input pattern differs from the target pattern. 


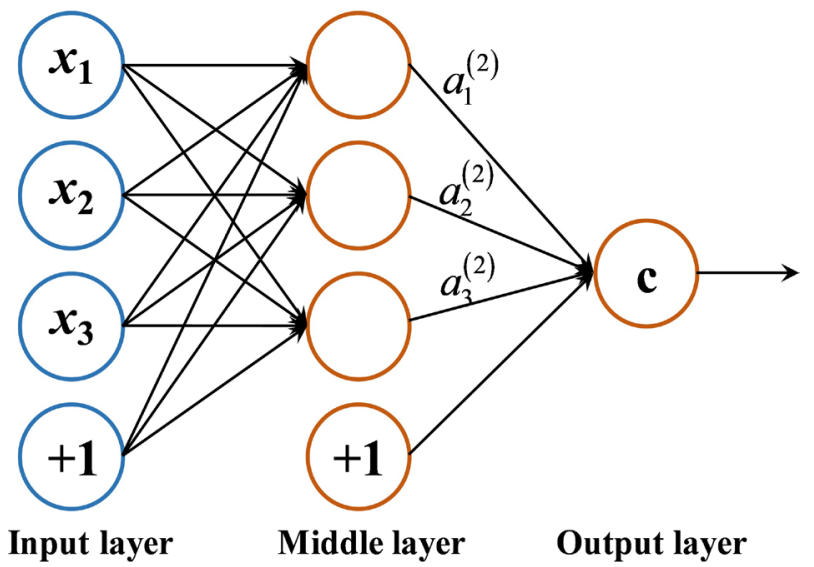

Figure 1. Schematic diagram of BP network. (The three hollow orange circles denotes three neurons of the middle layer, +1 in the last circle means the neurons can be added if required. The number of the neurons in the three layers can be added if required.)

One of the major problems with the BP algorithm is the slow convergence speed. A momentum factor is recommended by Lippmann to accelerate the convergence speed. Thus, Equation (4) can be converted into Equation (5).

$$
\Delta W_{i j}^{n}(T+1)=-\eta \frac{\Delta E}{\Delta W_{i j}^{n}}+\gamma^{*} \Delta W_{i j}^{n}(T)
$$

where $\gamma$ is the momentum factor and $\gamma \geq 0$. Once the partial derivative $E$ with respect to $W$ is obtained, it can be substituted into Equation (5) to arrive at the training formula for the adjustment of $W$ as

$$
\Delta W_{i j}^{n}(T+1)=-\eta \delta_{j}^{n} \frac{\Delta E}{\Delta W_{i j}^{n}}+\gamma^{*} \Delta W_{i j}^{n}(T)
$$

Similarly, the training formulae to adjust $\mathrm{t}, \beta$ and $\theta$ can be obtained as

$$
\begin{gathered}
\Delta t_{j}^{n}(T+1)=-\eta \delta_{j}^{n}+\gamma^{*} \Delta \delta_{j}^{n}(T) \\
\Delta \beta_{j}^{n}(T+1)=-\eta \xi_{j}^{n}\left[x_{j}^{n}\left(1-x_{j}^{n}\right) n e t_{j}^{n}\right]+\gamma^{*} \Delta \beta_{j}^{n}(T) \\
\Delta \theta_{j}^{n}(T+1)=-\eta \xi_{j}^{n}\left(x_{j}^{n} / \theta_{j}^{n}\right)+\gamma^{*} \Delta \theta_{j}^{n}(T)
\end{gathered}
$$

in which $\delta_{j}^{n}, \xi_{j}^{n}$ are adjustment coefficients.

\section{Modeling of the Network}

\subsection{Selection of the Characteristics of Pulse Manifestation}

Generally, $\mathrm{P}$ wave (main wave), tide wave, dicrotic wave in a cardiac cycle are often taken as the key characteristics of pulse manifestation. Sometimes the rates of change of these parameters are also chosen as the characteristics such as the maximum slop of the rising branch, slop 1 of the decent branch (the maximum slop between the main wave and the tide wave), slop 2 of the decent branch (the maximum slop between the tide wave and the dicrotic notch), the slop between the dicrotic wave and the end of the wave (Figure 2). 8 characteristics are chosen 


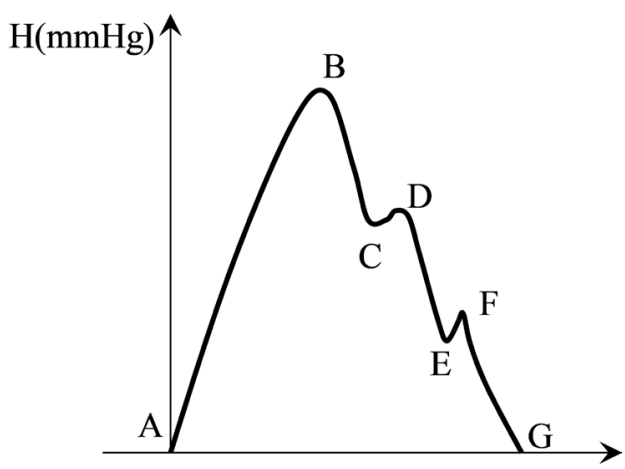

$\mathrm{T}(\mathrm{S})$

Figure 2. Schematic diagram of the characteristics of time domain in one cardiac cycle. ( $\mathrm{A}$ is the origin of the pulse in a circle, $\mathrm{B}$ denotes the highest point the pulse, $\mathrm{C}$ denotes the notch of the tide wave, $\mathrm{D}$ is the peak of tide wave, $\mathrm{E}$ is the dicrotic notch, $\mathrm{F}$ is the peak of dicrotic wave, $\mathrm{G}$ is the end of the pulse in a circle.)

in this study are as follows: the amplitude of the main wave (the value of the ordinate $B$ in Figure 2), the maximum slop in the rising branch (the maximum slop between $\mathrm{AB}$ in Figure 2) $\left(S_{a b}\right)$, slop 1 of the decent branch (the maximum slop between BC in Figure 2) $\left(S_{b c}\right)$, slop 2 in the decent branch (the maximum slop between DE in Figure 2) $\left(S_{d e}\right)$, amplitude of the dicrotic wave (the value of the ordinate $\mathrm{F}$ in Figure 2) $\left(H_{f}\right)$, amplitude of the dicrotic notch (the value of the ordinate $\mathrm{E}$ in Figure 2) $\left(H_{e}\right)$, cardiac cycle (the duration between AG in Figure 2) (T), ratio of amplitude of the dicrotic notch to cardiac diastole (the value of the ordinate E/duration between EG) $\left(R_{r}\right)$. For comparison, 6 characteristics and 4 characteristics (described in the following) are chosen from in the above characteristics to study.

The data in the following computing mainly come from the literatures [12] [13] [14] [15]. The characteristics in different literatures are slightly different, such as some gave the data of the amplitude of dicrotic notch while some gave the ratio of the amplitude of dicrotic notch to the amplitude of main wave. During the data preprocessing, the data are all converted to the amplitude of dicrotic notch. Meantime the data in the literature are very limited, some data are obtained by interpolation. The data of the normal pulse, slippery pulse, wiry pulse and thready pulse are adopted in this paper.

In the following analysis, three cases are considered to investigate the effects of different selections of characteristics: 1) The 8 characteristics described above; 2) Considering that the cardiac cycle and the amplitude of dicrotic notch can be obtained from the maximum slop in the rising branch, slop 1 in the decent branch, slop 2 in the decent branch, amplitude of dicrotic wave, the cardiac and the amplitude of dicrotic notch are not included in the second computing scheme; 3) In the third scheme, the slop 1 in the decent branch and the slop 2 in the decent branch are not considered.

The normal pulse, slippery pulse, wiry pulse and thready pulse are expressed by a four-dimensional array, respectively. That means, the output layer of the network has four neurons and each neuron has a value either 1 or 0 . The values 
of the four neurons of the output layer determine the type of the pulse manifestation: $\{1,0,0,0\}$ is corresponding to the normal pulse, $\{0,1,0,0\}$ the slippery pulse, $\{0,0,1,0\}$ the wiry pulse and $\{0,0,0,1\}$ the thready pulse.

\subsection{Selection of the Samples for Training and the Normalization of the Parameters}

For the same kind of pulse, the wave form may be very similar but the absolute value of the pulse's characteristics may be very different for different persons. Therefore, reasonable method is to adopt the dimensionless form of the characteristics in the network. According to the characteristics of the pulse manifestation, considering the value of each neuron of the network is between 0 - 1 for training and forecast easily, the minimum cardiac cycle $T_{m}$ of human in normal state is taken as the character time. The character pressure $H_{m}$ is determined by the requirement that the values of all the dimensionless slope in the data set should be less than 1.0. The characteristics in the data set can then be normalized by the character time and pressure. The dimensionless values of above 8 parameters are computed in the following way: main wave $\left(H_{b} / H_{m}\right)$, the maximum slop in the rising branch $\left(S_{a b} \times T_{m} / H_{m}\right)$, the slop 1 in the decent branch $\left(S_{b c} \times T_{m} / H_{m}\right)$, the slop 2 in the decent branch $\left(S_{d e} \times T_{m} / H_{m}\right)$, amplitude of dicrotic wave ( $\left.H_{f} / H_{m}\right)$, amplitude of dicrotic notch $\left(H_{e} / H_{m}\right)$, cardiac cycle $\left(T / T_{m}\right)$, amplitude of dicrotic notch/cardiac diastole $\left(R_{r} \times T_{m} / H_{m}\right)$. After such data processing, the network can be trained and used for forecast.

\section{Results and Discussion}

The network is trained by using 34 samples (shown in Table 1) in the data set first, and then is used to forecast by using 10 samples in the data set to test the accuracy of the network (shown in Table 2 are the data for forecasting with 8 characteristics).

During training of the network, it is required to trial many times by adjusting the parameters of the network till the accuracy is satisfied. For the data set shown in Table 1, the allowed total maximum error is adopted as 0.01 , the allowed single error is 0.001 , the maximum cycle number is 8000 , the learning rate is 0.7 , the coefficient of acceleration is 0.9 . One hidden layer is adopted whose neurons are two times of the input layer.

The results show that the accuracy of forecast can be over $85 \%$ when 8 characteristics of the pulse manifestation are chosen as the input of the network. The accuracy of forecast is about $60 \%$ when the network is input 6 characteristics and it is only $50 \%$ when selecting 4 input characteristics. Therefore, to select reasonable characteristics is the key to increase the forecast accuracy of the network. Generally, the more the selected characteristics are, the better the forecast effect is. Nevertheless, more characteristics often mean the measurement is more difficult and the cost is much more. So it is required to study how many and what characteristics are the most reasonable and meanwhile easy to measure for the neural network. 
Table 1. Data of pulse signal for training.

\begin{tabular}{|c|c|c|c|c|c|c|c|c|}
\hline $\begin{array}{c}H_{b} \\
10-2\end{array}$ & $\begin{array}{c}S_{b c} \\
10-2\end{array}$ & $\begin{array}{c}S_{d e} \\
10-2\end{array}$ & $\begin{array}{c}H_{f} \\
10-3\end{array}$ & $\begin{array}{c}H_{e} \\
10-3\end{array}$ & $T$ & $S_{a b}$ & $\begin{array}{c}R_{r} \\
10-2\end{array}$ & Type \\
\hline 4.31 & 15.87 & 9.19 & 4.59 & 15.26 & 0.46 & 63.41 & 3.21 & normal \\
\hline 1.68 & 1.46 & 2.92 & 1.2 & 1.17 & 0.53 & 1.69 & 0.14 & normal \\
\hline 8.13 & 46.7 & 26.27 & 13.16 & 52.87 & 0.98 & 19.85 & 6.45 & normal \\
\hline 2.39 & 6.13 & 5.25 & 2.15 & 2.63 & 0.57 & 3.21 & 0.73 & normal \\
\hline 3.11 & 10.8 & 7.88 & 3.59 & 4.98 & 0.61 & 4.67 & 1.34 & normal \\
\hline 6.08 & 12.84 & 18.97 & 2.99 & 36.46 & 0.90 & 56.04 & 6.71 & wiry \\
\hline 6.70 & 14.30 & 21.31 & 3.71 & 43.54 & 0.94 & 61.3 & 7.3 & wiry \\
\hline 7.42 & 16.05 & 23.35 & 4.31 & 51.17 & 1 & 66.26 & 8.03 & wiry \\
\hline 5.98 & 28.90 & 18.39 & 8.37 & 22.73 & 0.78 & 11.68 & 3.8 & normal \\
\hline 6.70 & 33.57 & 20.72 & 9.57 & 29.47 & 0.82 & 13.14 & 4.38 & normal \\
\hline 7.42 & 40.86 & 23.35 & 10.77 & 37.08 & 0.86 & 14.59 & 4.96 & normal \\
\hline 4.98 & 16.05 & 35.61 & 0.65 & 23.92 & 0.90 & 97.49 & 4.7 & wiry \\
\hline 4.88 & 39.99 & 26.56 & 4.67 & 13.13 & 0.94 & 99.53 & 3.85 & slippery \\
\hline 2.75 & 6.71 & 22.48 & -0.14 & 12.73 & 0.63 & 50.5 & 3.36 & thready \\
\hline 0.43 & 1.46 & 1.46 & 0 & 0.78 & 0.44 & 11.68 & 0.91 & thready \\
\hline 3.35 & 11.68 & 8.76 & 11.96 & 18.62 & 0.75 & 32.11 & 4.17 & thready \\
\hline 0.72 & 2.48 & 2.19 & 1.2 & 1.58 & 0.48 & 13.72 & 1.23 & thready \\
\hline 1.01 & 3.50 & 2.92 & 2.39 & 2.63 & 0.51 & 15.76 & 1.55 & thready \\
\hline 1.29 & 4.52 & 3.65 & 3.59 & 3.88 & 0.54 & 17.81 & 1.87 & thready \\
\hline 1.58 & 5.55 & 4.38 & 4.78 & 5.36 & 0.57 & 19.85 & 2.19 & thready \\
\hline 2.73 & 9.63 & 7.30 & 9.57 & 12.68 & 0.70 & 28.02 & 3.47 & thready \\
\hline 3.01 & 10.65 & 8.03 & 10.77 & 16.99 & 0.74 & 30.06 & 3.8 & thready \\
\hline 2.03 & 1,46 & 1.46 & -1.67 & 4.88 & 0.39 & 20.43 & 1.46 & thready \\
\hline 8.13 & 17.51 & 26.27 & 4.78 & 59.14 & 1 & 70.05 & 8.52 & thready \\
\hline 2.63 & 3.07 & 3.94 & 0.96 & 7.89 & 0.49 & 25.39 & 2.19 & thready \\
\hline 2.15 & 2.92 & 1.46 & 0 & 1.51 & 0.44 & 26.27 & 0.47 & slippery \\
\hline 8.61 & 27.73 & 20.43 & 11.96 & 34.88 & 0.82 & 96.32 & 5.72 & slippery \\
\hline 2.87 & 5.34 & 3.3 & 1.2 & 2.96 & 0.48 & 33.28 & 0.99 & slippery \\
\hline 3.47 & 7.88 & 5.2 & 2.63 & 4.86 & 0.52 & 37.36 & 1.52 & slippery \\
\hline 4.07 & 10.51 & 7.06 & 3.83 & 6.91 & 0.55 & 44.367 & 2.04 & slippery \\
\hline 4.74 & 12.99 & 8.96 & 4.78 & 9.57 & 0.59 & 51.37 & 2.86 & slippery \\
\hline 5.38 & 15.47 & 10.8 & 5.98 & 12.54 & 0.62 & 58.38 & 3.39 & slippery \\
\hline 5.98 & 18.1 & 12.7 & 7.3 & 15.91 & 0.66 & 65.38 & 3.91 & slippery \\
\hline 6.63 & 20.58 & 14.59 & 8.37 & 19.88 & 0.7 & 72.39 & 4.44 & slippery \\
\hline 5.5 & 11 & 16.3 & 2.15 & 30.3 & 0.80 & 0.51 & 5.84 & wiry \\
\hline 1.9 & 6.6 & 5.11 & 5.98 & 7.18 & 0.61 & 0.22 & 2.51 & thready \\
\hline
\end{tabular}




\begin{tabular}{|c|c|c|c|c|c|c|c|c|}
\hline \multicolumn{9}{|c|}{ Continued } \\
\hline 2.2 & 7.6 & 5.84 & 7.18 & 9.04 & 0.64 & 0.24 & 2.83 & thready \\
\hline 2.4 & 8.6 & 6.57 & 8.37 & 11.24 & 0.67 & 0.26 & 3.15 & thready \\
\hline 3.8 & 6.3 & 8.82 & 1.34 & 17.15 & 0.70 & 0.34 & 3.85 & thready \\
\hline 3.8 & 15.5 & 10.5 & 4.78 & 7.66 & 0.66 & 0.06 & 1.96 & normal \\
\hline 4.5 & 19.8 & 13.14 & 5.98 & 11.8 & 0.70 & 0.08 & 2.54 & normal \\
\hline 5.3 & 24.2 & 15.76 & 7.18 & 16.84 & 0.74 & 0.10 & 3.21 & normal \\
\hline 7.25 & 23.06 & 16.65 & 9.6 & 24.14 & 0.73 & 0.79 & 4.96 & slippery \\
\hline 7.9 & 25.69 & 18.54 & 10.77 & 28.82 & 0.77 & 0.86 & 5.49 & slippery \\
\hline
\end{tabular}

Table 2. Data of pulse signal for forecast.

\begin{tabular}{|c|c|c|c|c|c|c|c|c|c|}
\hline $\begin{array}{c}H_{b} \\
10-2\end{array}$ & $\begin{array}{c}S_{b c} \\
10-2\end{array}$ & $\begin{array}{c}S_{d e} \\
10-2\end{array}$ & $\begin{array}{c}H_{f} \\
10-3\end{array}$ & $\begin{array}{c}H_{e} \\
10-3\end{array}$ & $T$ & $S_{a b}$ & $\begin{array}{c}R_{r} \\
10-2\end{array}$ & $\begin{array}{c}H_{b} \\
10-2\end{array}$ & $\begin{array}{c}S_{b c} \\
10-2\end{array}$ \\
\hline 5.5 & 11 & 16.3 & 2.15 & 30.3 & 0.80 & 0.51 & 5.84 & wiry & wiry \\
\hline 1.9 & 6.6 & 5.11 & 5.98 & 7.18 & 0.61 & 0.22 & 2.51 & thready & normal \\
\hline 2.2 & 7.6 & 5.84 & 7.18 & 9.04 & 0.64 & 0.24 & 2.83 & thready & thready \\
\hline 2.4 & 8.6 & 6.57 & 8.37 & 11.24 & 0.67 & 0.26 & 3.15 & thready & normal \\
\hline 3.8 & 6.3 & 8.82 & 1.34 & 17.15 & 0.70 & 0.34 & 3.85 & thready & thready \\
\hline 3.8 & 15.5 & 10.5 & 4.78 & 7.66 & 0.66 & 0.06 & 1.96 & normal & normal \\
\hline 4.5 & 19.8 & 13.14 & 5.98 & 11.8 & 0.70 & 0.08 & 2.54 & normal & normal \\
\hline 5.3 & 24.2 & 15.76 & 7.18 & 16.84 & 0.74 & 0.10 & 3.21 & normal & normal \\
\hline 7.25 & 23.06 & 16.65 & 9.6 & 24.14 & 0.73 & 0.79 & 4.96 & slippery & slippery \\
\hline 7.9 & 25.69 & 18.54 & 10.77 & 28.82 & 0.77 & 0.86 & 5.49 & slippery & slippery \\
\hline
\end{tabular}

\section{Conclusions}

Pulse diagnosis, one of the four diagnostic methods of traditional Chinese medicine, is always constrained by the human subjective factors. Therefore, the objectification of the pulse analysis attracts the attention of researchers. The classification of the pulse manifestation is studied by using the BP artificial neural network. The network is modeled by measured data. It has been studied on how to select the characteristics of the pulse manifestation and how to preprocess the data. A method of data preprocessing on how to nondimensionalize and normalize the characteristics of pulse manifestation is presented. It is shown that the data after preprocessing are more suitable for the training of the network and the increase of the forecast accuracy.

The effects of the selection of different characteristics on the classification of pulse manifestation are investigated. It is shown that the forecast accuracy increases with the increase of characteristic number.

Although the data adopted is not very much enough and so the results are limited, this study shows that the BP neural network is an effective tool for the identification of the pulse manifestation. 


\section{Conflicts of Interest}

The authors declare no conflicts of interest regarding the publication of this paper.

\section{References}

[1] Yang, L.J. (2006) Study on the Pulse Analysis System and Identification of Pulse Signal by Artificial Neural Network. Master's Thesis, Northeast Forestry University, Harbin.

[2] Yang, B., Niu, X. and Wang, Y.L. (2000) Advances of the Research on the Pulse Diagnosis Instrument and Analysis Method. Journal of Beijing University of Traditional Chinese Medicine, 23, 68-69.

[3] Yang, S. (2017) Research of Pulse Signals Extraction and Method Based on Cluster Analysis of the Pulse Manifestation. Master's Thesis, Harbin Institute of Technology, Harbin.

[4] Wang, B.H. (1999) Study of Human Pulse System Modelling and Pulse Information Analysis. Ph.D. Thesis, Northwest Polytechnical University, Xi'an.

[5] Yan, H.X., Wang, Y.Q., Gong, A.M., et al. (2009) Application of the Artificial Neural Network in Recognition and Classification of TCM Pulses. World Science and Techology/Modernization of Traditional Chinese Medicine and Material Medica, 11, 522-526.

[6] Wang, L., Wang, K.Q., Xu, L.S., et al. (2005) Classifier of Pulse Manifestation Based on the Fuzzy Neural Network. Collection of Papers on the Application and Research of Computers in Diagnose, 84-90.

[7] Guo, H.X., Wang, B.H., Zhang, L.Q., et al. (2006) The Identification Method of Pulse Manifestation Based on Wavelet Package and BP Neural Network. Application Research of Computers, 6, 185-187.

[8] Zhang, J. (2010) The Research of Human Body Pulse Signals Extraction and Classification. Master's Thesis, Soochou University, Suzhou.

[9] Anderson, J.A. (1995) An Introduction to Neural Networks. MIT Press, Cambridge, MA. https://doi.org/10.7551/mitpress/3905.001.0001

[10] Fuh, K.H. and Wang, S.B. (1997) Force Modeling and Forecasting in Creep Feed Grinding Using Improved BP Neural Network. International Journal of Machine Tools and Manufacture, 37, 1167-1178. https://doi.org/10.1016/S0890-6955(96)00012-0

[11] Lu, X.B. and Li, D.J. (1996) Application of BP Network in the Study of Debris Flow. Chinese Journal of Rock Mechanics and Engineering, 15, 766.

[12] Chen, S.Y., Wang, S.Y., Lv, Z.P., et al. (1985) Analysis of the 70 Figures of Wiry Pulse Manifestation. Journal of First Military Medical University, 5, 57-59.

[13] Chen, S.Y. and Wang, S.Y. (1982) Analysis of the 110 Figures of Normal Pulse Manifestation. Journal of First Military Medical University, 2, 332-334.

[14] Xiao, G., Li, L.Y., Li, G.H., et al. (1980) Analysis of the 188 Figures of Abnormal Pulse Manifestation. Journal of Shandong Medical College, 4, 28-36.

[15] Xiao, G., Li, L.Y., Li, G.H., et al. (1980) Analysis of the 212 Figures of Normal Pulse Manifestation. Journal of Shandong Medical College, 4, 23-27. 